\title{
Modeling Zombie Outbreaks: A Problem-Based Approach to Improving Mathematics One Brain at a Time
}

\begin{abstract}
A great deal of educational literature has focused on problembased learning $(\mathrm{PBL})$ in mathematics at the primary and secondary level, but arguably there is an even greater need for PBL in college math courses. We present a project centered around the Humans vs. Zombies moderated tag game played on the USU campus. We discuss the project in the context of an undergraduate differential equations course and discuss how the project is launched. We highlight examples of students mathematical models along with their verbal and written responses as well as discussing assessment and student learning. Results are discussed in the context of higher and lower cognition levels as well as mathematical appreciation.
\end{abstract}

Keywords: problem-based learning, modeling, logistic equation, differential equations, zombies

\section{Introduction}

Mastering mathematics is similar to learning a language. When acquiring a new language, classes can be very helpful to teach the basic vocabulary and grammar. Similarly, in mathematics classes students learn rules, notation, techniques and algorithms analogous to grammar and sentence structure in languages. However, as students of language know, time spent using the language in the real world brings about a fluency that cannot be fully fleshed out in a typical classroom setting.

While it is important for students to be well-versed in mathematical techniques it is clear that students are currently trained to have a 
rigorous knowledge of mathematical grammar but are barely conversational and certainly not colloquial when they 'speak' mathematically [8]. Students that are 'conversational' in mathematics are able to use mathematics to describe the world around them. Students should be able to understand why the various terms are a part of a mathematical model and produce a physical interpretation of the terms. Additionally, mathematically conversational students should be able to assess the task at hand and use a variety of mathematical approaches, techniques and skills they have acquired (sometimes in unconventional ways) to accomplish the goal.

To broaden the student experience, textbooks contain a plethora of contrived problems. While these exercises help students polish the basic skills, they often do not increase students' mathematical language fluency beyond the typical exercise found in the text. Often students are told the exact model to use and how they should implement it. The model is also often presented as the only correct one, removing the opportunity for a student to consider whether it is appropriate or not. However, in mathematics, as in language, there are a variety of styles and methods to describe the same phenomenon.

In an effort to help students become more mathematically fluent there has been a surge in problem-based learning (PBL), a "teacherfacilitated, student-driven approach" [3], since learning through discovery is the way conversational math skills are acquired $[2,4,11]$. Although PBL has become more prevalent in elementary and secondary school, in the college classroom it is largely confined to medical and engineering curricula and is far less visible in college mathematics classrooms [1]. When a mathematics course does have an inquiry-based module it often consists of canned data for which a predetermined model fits well. This undermines the creative and fluency-building potential of the project.

By contrast, when students are involved in the data collection process, they have a more intuitive understanding of the mechanisms driving the data [10]. This motivates modeling the data and makes it more clear to students when their models are successful. Generating and describing data gives students a sense of responsibility and produces useful learning 
lessons, provided the instructor properly designs the exercise.

We present a modeling experience for college differential equations called the Zombie Lab, which is a data-driven activity centered around the Humans vs Zombies (HvZ) game played on numerous college campuses [13]. We outline the materials used to set the lab in motion as well as discuss some of the techniques and approaches used by students. To highlight the efficacy of the lab, we also provide assessment questions that target higher learning levels and discuss student performance along with student responses. Ultimately, we argue that PAVE provides college mathematics students with the opportunity to achieve deeper learning and better mathematical fluency.

\section{Materials and Methods}

\subsection{Launching the Zombie Lab}

\subsubsection{Basic Zombie Game}

To introduce disease dynamics and get students started connecting models with data, the lab begins with a simple human/zombie simulation following the outline of the Basic Disease Game [8]. Class members simulate zombies attacking and infecting a human population. The game is played using two transparencies filled with 100 adjacent hexagons (Figure 1). Students are divided into groups of 3 or 4 , and one team member directs the zombie population (Zombie Master), one leads the human population (Humanoid King/Queen) and one judges results and records data. Zombies and humans are placed by their respective rulers on separate hex transparencies using dry-erase markers of different colors beginning with 1 zombie and 49 humans. For the basic Zombie Game each zombie occupies three consecutive hexes, one for its huge head and one for each of its attacking arms, while humans fill only one hex each. Once the Zombie Master and Humanoid King/Queen have secretly placed their respective players on the hex arenas, one determines which humans have been successfully attacked by counting the hexes that are occupied by a human and a zombie arm or head when the transparencies 
are superimposed. These new zombies are added to the zombie population and transparencies are then erased for the subsequent turn. The game is over once the entire population has been zombified.

To set the stage for the rest of the class, we focus on the primary example of a population model found in most undergraduate differential equations textbooks, Pierre-François Verhulst's logistic population model [12]. Let $Z$ represent the zombie population and $K$ be the population's carrying capacity. Then $1-\frac{Z}{K}$ would be the probability of

encountering a non-zombie and thus, $Z\left(1-\frac{Z}{K}\right)$ would be proportional to the expected number of zombie, non-zombie contacts. This leads to the logistic model

$$
\frac{d Z}{d t}=\lambda Z\left(1-\frac{Z}{K}\right)
$$

where $\lambda$ represents the rate at which non-zombies are aggressively converted to zombies. Since there are only 50 total humans and zombies in the Basic Zombie Game, we set $K=50$. After solving analytically, the model is fit to the Basic Zombie Game data in the typical textbook fashion - pick a data point, plug it into the solution of the differential equation and solve for $\lambda$. When plotted alongside the data from the Basic Zombie Game, it is evident that while the model is not perfect, it does match the general shape of the data (Figure 2). To justify the shortcomings of the model, students tend to initially blame themselves for collecting 'bad' data or using bad parameters. However, when discussed as a whole class, students quickly conclude that the model is not built to adequately capture the entire dynamics of the Basic Zombie Game. The instructor can then lead the class in a discussion of possible improvements and alternative models.

\subsubsection{Humans vs. Zombies}

With students primed for modeling from the Basic Zombie Game, we introduce the data collected from the Humans vs. Zombies (HvZ) game played on campus. HvZ is a game of moderated tag that started at Goucher College in 2005 and is currently played on campuses worldwide 


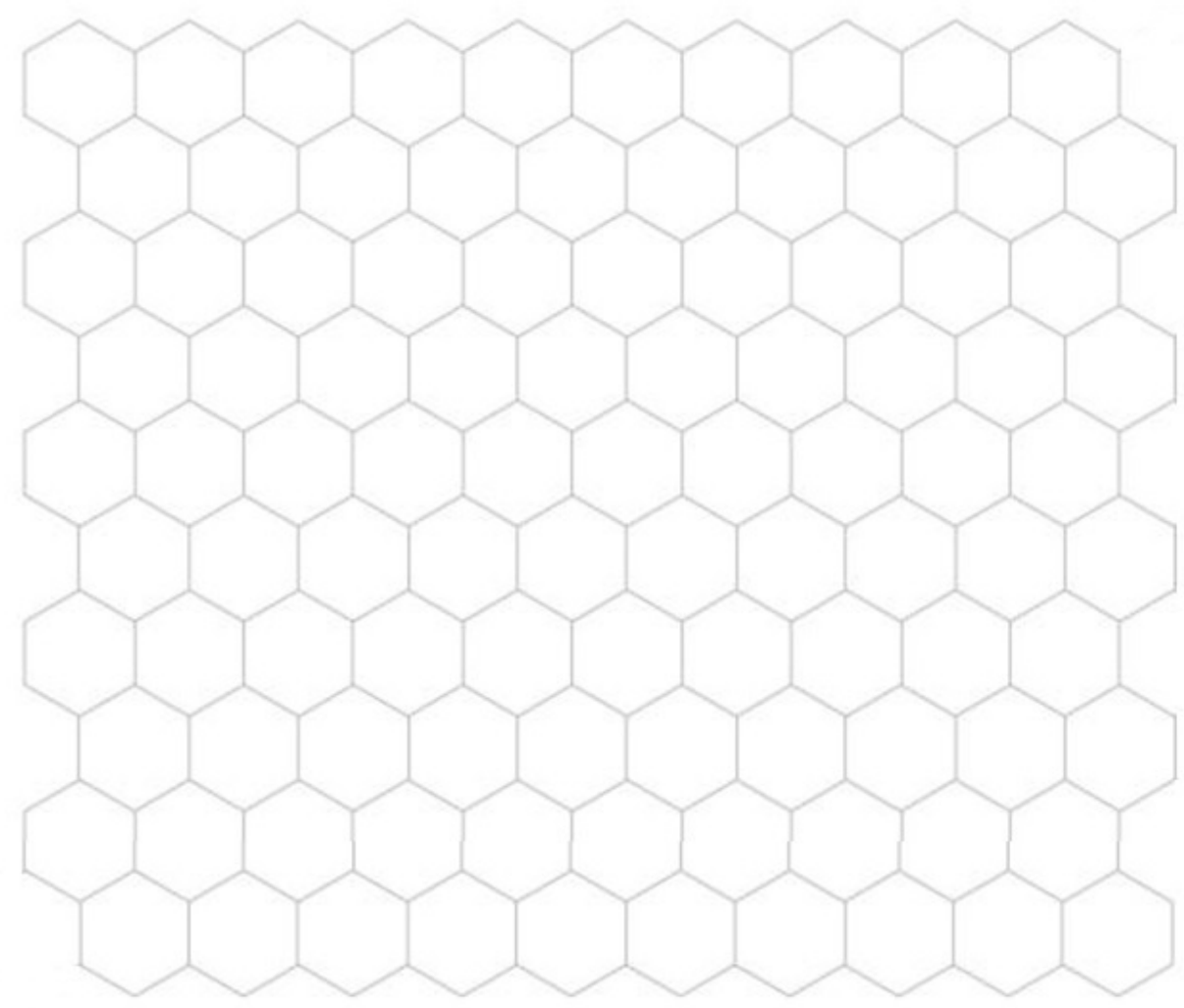

Figure 1. The hex transparency for the Basic Zombie Game used to simulate how zombies and humans may interact. To play the game, two participants have separate transparencies and one player situates the humans on his/her transparency while the other player marks the hexes affected by zombies. When the transparencies are then superimposed, humans in a zombie affected space are zombified and added to the zombie population. 


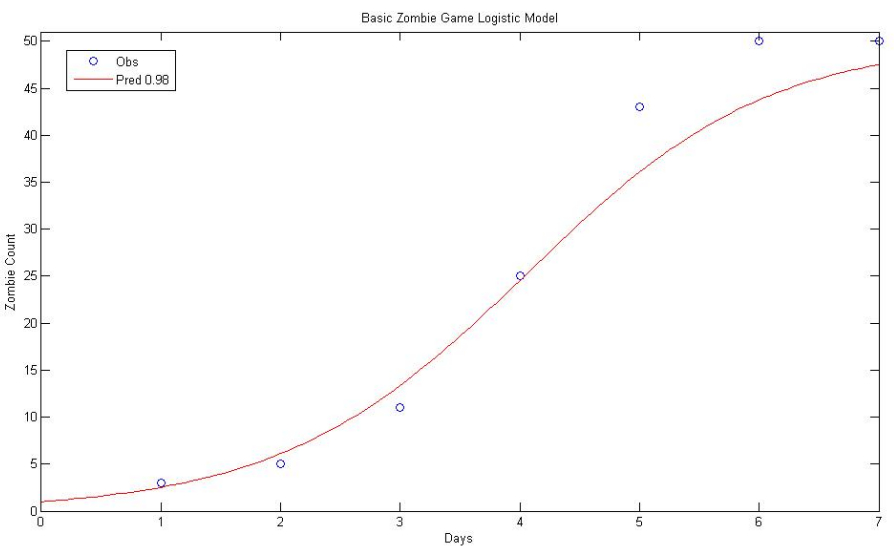

Figure 2. Sample data from the Basic Zombie Game along with the predicted zombie population generated from the Logistic Model. The solid curve $(\lambda \approx$ 0.98 ) is parameterized by substituting data at time $t=4$ when the zombie population $Z=25$. Note that the model performs arguably well initially and then dramatically decreases in accuracy after day 4 when the zombie population is about half of the total population of 50 . 
[13]. The game is played in a bounded area at specific hours of the day, e.g. on campus from 8 a.m. to 10 p.m., excluding buildings. Humans are converted into zombies by touch alone. Humans can defend themselves by stunning zombies for 15 minutes with a Nerf dart gun or by pelting zombies with a pair of socks rolled up into a ball. Also, a zombie dies if it does not infect a human within a 24 hour period. Additionally, humans are required to fulfill certain missions at various points during the game. These missions result in large fatalities in the human population and a corresponding increase in the zombie population. In order to track the progress of the game, zombies are required to report the ID number of each human they tag. This data drives the second portion of the Zombie Lab.

In general, students are excited to see this data since they have all played or witnessed the HvZ game on campus. It is an engaging set of data that fits the students' observations of the game. For most students it is immediately clear that the data does not resemble the data created in the Basic Zombie Game and that the Logistic Model will not be an effective model. When assigned the task of developing a model for the HvZ data, students easily recognize the authenticity of the effort. The lab is original - nobody has previously solved this problem and the data set is not the result of an instructor's calculated efforts. Additionally, since there is no single correct answer, there is ample opportunity for students to explore and connect concepts as needed. It promotes discovery. Since the students plot their models with the data, they are able to visibly distinguish how successful their models are.

\subsubsection{Student Expectations and Lab Agenda}

The general objectives for student groups are:

1. Create a model which will predict the zombie and human populations of the HvZ game.

2. Estimate parameters using data and model hypotheses.

We ask the groups to produce a short paper (less than 5 pages). The paper must contain: 


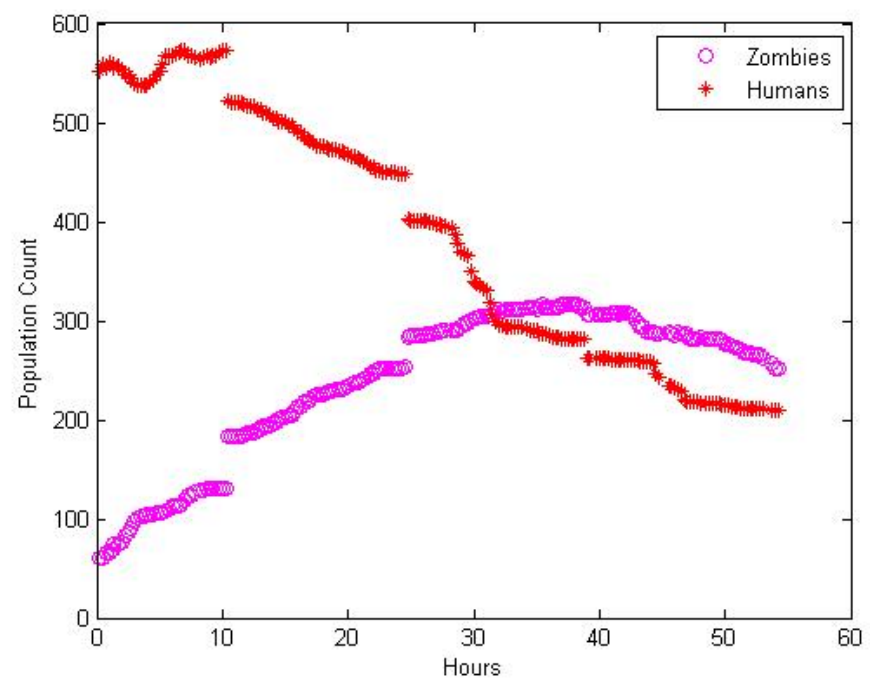

Figure 3. Data from the Humans vs. Zombies game played on the USU campus depicting the rise and fall of the zombie population (o) in relation to the human population $(*)$ over time. New players were allowed to join the game during the first day (hence the increases in the human population). Additionally, the jumps in the populations were due to missions the humans were required to fulfill at various points in the game that result in many humans being turned to zombies.

1. An introduction describing the problem and its significance,

2. A methods section that contains a description and justification of their proposed HvZ model and a clear explanation of how parameters were estimated,

3. A results section that describes how well the HvZ model performed with as well as a picture of the predicted populations plotted with the HvZ data for a visual reference,

4. A discussion and conclusion section detailing model implications. 
It is required that each team member assumes responsibility for some portion of the report but students must work together in order to ensure that one team member's section flows seamlessly into the next. The lab reports are graded on clarity, consistency, grammar and presence of required elements.

For a class of 20 - 30 students the Zombie Lab's general outline is:

- (Lecture) Introduction to Zombie Lab and Basic Zombie Game (15 minutes)

- (Data Collection) Group Work: Designate roles for Basic Zombie Game and play game at least 2 times (15 minutes)

- (Lecture) Derivation of Logistic Model (20 minutes)

- (Model Construction) Group Work: Using graphing utility (e.g. Excel, Matlab, TI-89) to plot Basic Zombie Game data, calculate $\lambda$ and plot the fitted Logistic Model (30 minutes)

- (Model Construction/Data Collection) Class Discussion: Groups compare Logistic Model results and share ideas on how to improve the model (10 minutes)

- (Lecture) Introduction to HvZ game and data (10 minutes)

- (Model Construction/Task Assignment) Group Work: Groups develop a model for the HvZ data and determine which portions of the report each will be responsible for (45 minutes)

- (Model Presentation) Class Discussion: Groups present models for HvZ data including description of units parameters (40 minutes)

In all, the project takes $3-4$ hours of class time or about one week during the semester. Naturally, this schedule can be tightened (e.g. $\lambda$ can be calculated as a class) as the instructor needs and is aimed to be accomplished over the span of multiple class periods. Between class days, students are expected to meet regularly as groups to further develop their models and compare with data. 


\section{Student Models}

To facilitate the student learning experience it is helpful if instructors are aware of strategies employed by students. We typically situate the Zombie Lab early in the semester of an ordinary differential equations course immediately after the section in our text, [5], about Euler's Method. By scheduling the lab early we emphasize the importance of developing mathematical fluency through modeling. Typically, the lab occurs around the fourth week of the class when students have a few analytic solution techniques (methods of separation of variables and integrating factor) as well as the newly learned numerical approach. When interacting with students during the Zombie Lab, particular care is taken to scaffold student thinking, or to "use leading questions to guide the reasoning and direction of the conversation, yet allow students to make connections themselves" [9]. This challenges students to make greater connections with the classroom materials and previous classes while ensuring high cognitive performance. Following are a few examples from two undergraduate differential equations courses, one with 28 students and the other with 24 students, who participated in the lab in Spring 2013.

\subsection{Active Participants Model}

A major flaw students see with modeling the HvZ data with Verhulst's Logistic Model is that it does not allow the zombie population to decrease as seen in figure 3. A typical approach is to incorporate zombie death into equation (1) leading to

$$
\frac{d Z}{d t}=\alpha H Z-\beta Z
$$

where $\beta$ is the zombie death rate and $\alpha$ is the zombie population infection

rate. In one group, the students hypothesized from their experience playing the game that many of the participants that were caught early would simply quit the game and according to the rules of the game, starve to death 24 hours later. They did not participate as zombies. This led them to conjecture that there is a constant population of core 
players that participate from the beginning to the end. They focused on this constant core group, $T$, called active participants, and ignored the rest of the players hypothesizing that the "... quickly disappearing, nonactive players will only cause a little error in the model's predictions at the beginning." Hence, they let the constant $T=H+Z$ or $H=T-Z$ and substituted into (2). The students then had

$$
\frac{d Z}{d t}=\alpha[(T-Z) Z-\xi Z]
$$

a separable differential equation where $\xi=\beta / \alpha$. In order to create the model, students used what they knew from the derivation of the Logistic Model and altered it to meet their primary concern of how to account for zombie death.

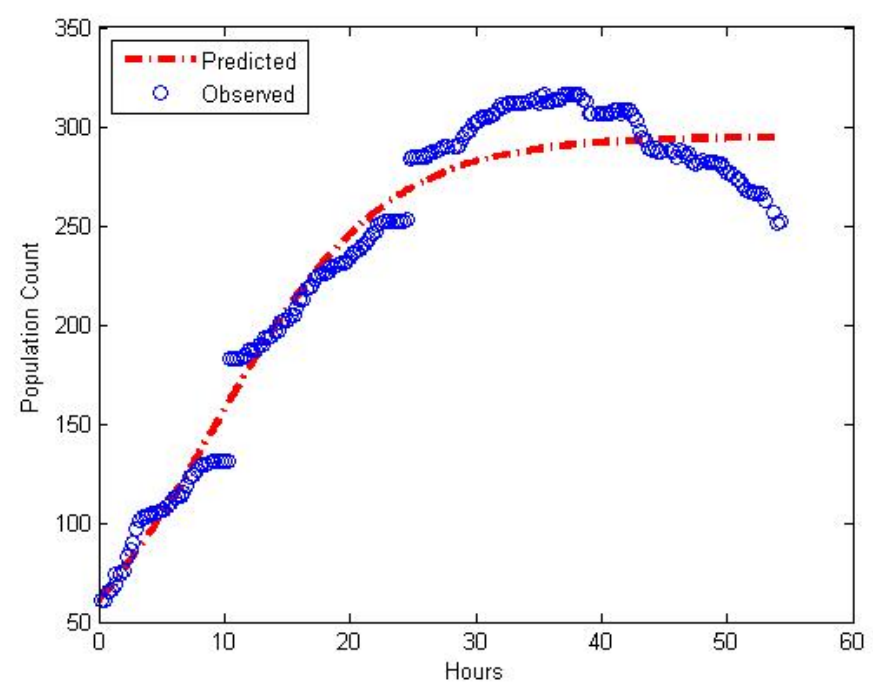

Figure 4. Plot of the Active Participants student model fitted to the HvZ zombie population data using least squares approximation. Students estimated the population growth rate $\alpha \approx 0.0005$ and $\alpha / \beta=\xi \approx 5$ where $\beta$ is the zombie death rate. The model is based on the hypothesis that players often quit the game once they're turned to zombies which increases zombie mortality. 
With a model that fit their hypotheses in hand, the students set out to estimate their parameters. Some students in the group had taken a linear algebra course and knew that using least-squares approximation was an option, but did not know how to implement it. After a short discussion with the instructor and some help from online resources they determined parameters that fit the data well. While this is a sizable deviation from from the textbook pick-a-point method, it is a typical practice used in both academic research and industry alike. When the students plotted their parameterized model with the data (Figure 4), they were immediately able to conclude that they were not $100 \%$ successful. In particular, they noted how their model's peak, timing and end behavior were not in line with the data. In the conclusion of their written report, the students mentioned how the model adequately duplicates the initial zombie population growth and conjectured that developing a non-constant model for the active participants would greatly improve the model.

\subsection{Threshold Model}

Another group conjectured that the rate of zombies dying or simply quitting the game would grow with time due to the increasingly scant supply of humans to feed on as the game progressed as well as zombified students simply quitting when the action died down a bit. Additionally, they supposed that there is a critical zombie threshold population, $P$, that if crossed would cause a collapse of the zombie population. In order to accommodate these two hypotheses the students' model took the following form:

$$
\frac{d Z}{d t}=-r\left(1-\frac{Z}{P}\right)\left(1-\frac{Z}{K}\right) Z-s t Z
$$

where $r$ is the intrinsic growth rate, $K$ is the carrying capacity and $s$ describes the increasing rate at which zombies die or leave the game. Even though the model the students developed could not be solved with the analytic methods that had been taught at this point in the course, the students were excited to utilize the recently learned Euler method 
to solve their new problem. This particular model is based on classroom and textbook examples that the students creatively adjusted to fit their needs. While the model's success is primarily driven by the $-s t Z$ term, in the end, the students produced a data-driven, mechanistic model that reflects their hypotheses.

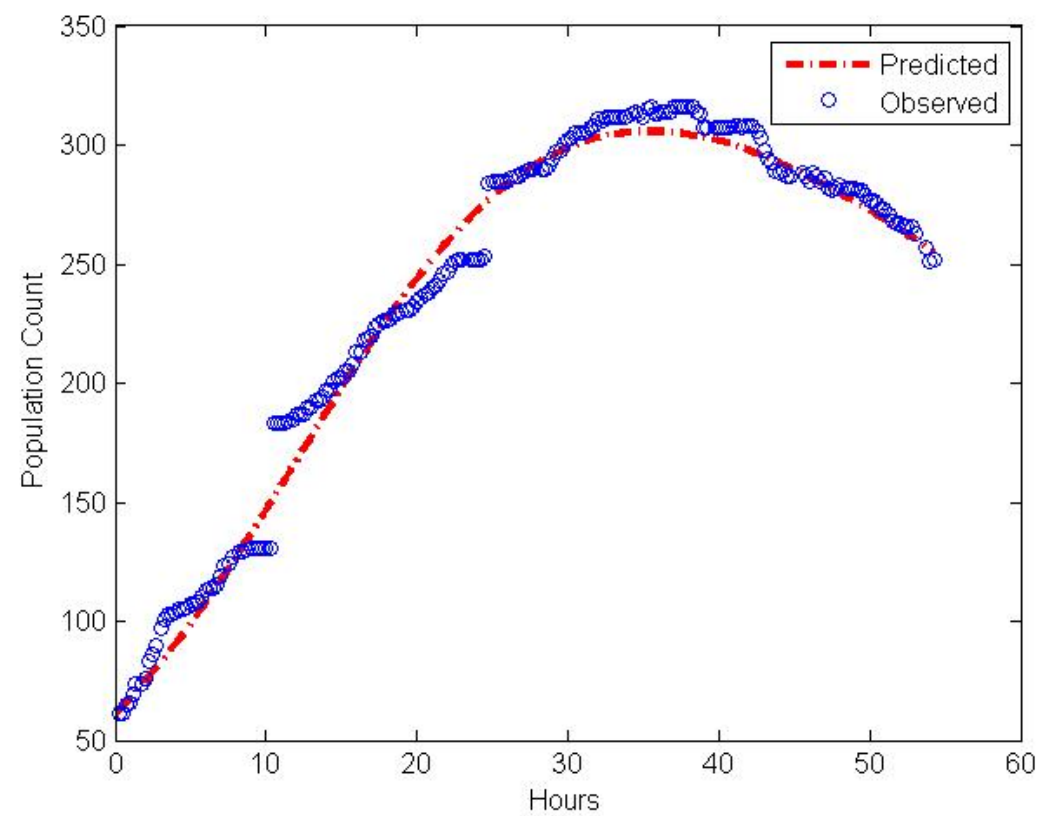

Figure 5. Plot of students' Threshold Model fitted to the HvZ zombie population data using least squares. Students estimated the intrinsic growth rate at 0.122 and the death acceleration term $s \approx 0.0014$. The model is based on the hypothesis that once the zombie population has been reduced below a critical threshold it would naturally collapse to zero; students did not notice that their threshold, $P \approx 840$, was very similar to the corresponding $K \approx 845$.

Similar to the previous model, students used least squares approximation to determine their parameters. As seen in figure 5, the students' model closely matches the HvZ data. However, in the students' written report, they were quick to point out that their model fails to capture 
the large jumps around the 10 and 25 hour mark. They went on to explain how their model was not designed to capture the missions that the humans were required to perform at those points in the game. They then conjectured that for future zombie research they would like to parameterize and define their model piece-wise on the $[0,10],(10,25]$ and $(25,55]$ hour intervals separately to better account for the missions.

\subsection{The Answer's a Parabola, Right? Model}

Some students have had experience fitting polynomial curves to data, and when confronted with the HvZ data (figure 3) immediately want to fit a parabola. Most of these students are initially straightforward with their intentions, suggesting models of the form $\frac{d Z}{d t}=A t+B$ where $A$ represents the population's rate of acceleration and $B$ is the growth rate. Many realize their approach is off target when asked to either describe the relationships between the variables they used to create the model or outline the physical concepts upon which the model was derived. They come to understand the models are supposed to be mechanistic and not strictly empirical models used to drive a curve through points. However, others remain determined that the parabola is the right answer, but it simply needs to be dressed up more.

In one class, the lab occurred shortly after the students had learned the method of integrating factors. In one homework assignment students were asked to "...construct a first order linear differential equation whose solutions have the required behavior as $t \rightarrow \infty$ " [5]. The students were then assigned a variety of functions their solutions should approach. Inevitably, some students noted they could simply extend their homework experience to the HvZ scenario and produced the model $\frac{d Z}{d t}+Z(t)=\frac{d g}{d t}+g(t)$ where $g(t)=A t^{2}+B t+C$, the parabola the students want to use to model the data. The students proceeded to solve the differential equation using the method of integrating factors to get $Z(t)=A t^{2}+B t+C+D e^{-t}$ (figure 6), a function that approaches $g(t)=A t^{2}+B t+C$ asymptotically.

So, while every student model will not be mechanistic, the students 


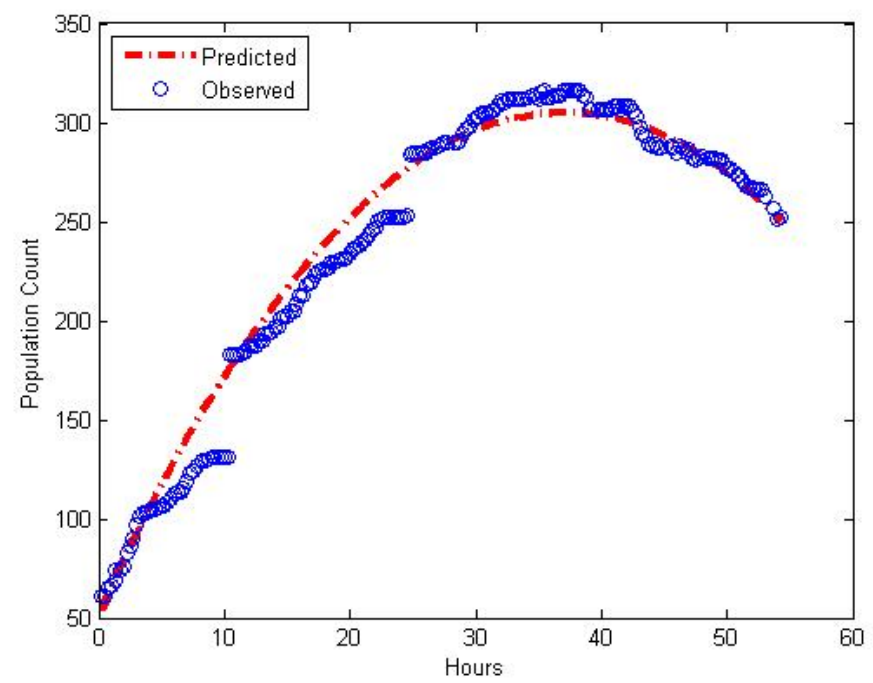

Figure 6. Plot of The Answer's a Parabola, Right? student model. Students constructed a differential equation whose solution asymptotically approached $g=54+13.55 t-0.18 t^{2}$, the quadratic fit.

were using mathematics to explain and describe data fluently which is seldom seen in a typical differential equations classroom.

\section{Assessment of the Zombie Lab}

We are interested in assessing how well the students who participated in the Zombie Lab achieve objectives in a variety of learning levels defined in [6]. These include:

- Higher Cognitive Load

- Construct-a-Concept: Students achieve an objective at the constructaconcept learning level by using inductive reasoning to distinguish examples of a particular concept from nonexamples of that concept.

- Discover-a-Relation: Students achieve an objective at the discoverarelationship learning level by using inductive reason- 
ing to discover that a particular relationship exists or why the relationship exists.

- Comprehension-and-Communication: Students achieve an objective at the comprehensionandcommunication level by (i) extracting and interpreting meaning from an expression, (ii) using the language of mathematics, and (iii) communicating with and about mathematics.

- Creative-Thinking: Students achieve an objective at the creativethinking learning level by using divergent reasoning to view mathematical content from unusual and novel ways.

- Lower Cognitive Load

- Algorithmic Skill: Students achieve an objective at the algorithmicskill level by remembering and executing a sequence of steps in a specific procedure.

- Affective Domain

- Appreciation: Students achieve an objective at the appreciation learning level by believing the mathematical content specified in the objective has value.

Naturally, our focus in designing the lab is to draw from learning areas (e.g., Comprehension-and-Communication, Discover-a-Relation, etc.) that are difficult to reach in a traditional lecture setting. However, other areas of cognition (e.g., Algorithmic Skill) are intrinsically embedded within the lab's structure. While these are important areas of learning, we did not target them specifically in the assessment since they can be easily assessed from other coursework. By using the Zombie Lab to complement the traditional coursework we hope to target mathematical fluency more efficiently.

While it's tempting to create lengthy formal assessments to measure every possible aspect of student learning occurring in the lab, it is far more important to focus the limited class time on learning/teaching by picking a few objectives and learning levels to target in assessment. A short (5 questions) pre- and post-test focused on course objectives at a 
variety of learning levels was designed and given to the students the first and last days of the lab (see Appendix A).

The first three questions (Microsoft per-capita hiring, data arrangement, rabbit per-capita growth) deal with different areas of model development. In the Microsoft question students are expected to determine whether the given per-capita data implies exponential growth. The second question asks students to explain how they might rearrange data to generate ideas for models. The third question asks students to develop a population model given per-capita growth data of a rabbit population. The fourth question (Gompertz model) directs students to find equilibrium solutions and sketch solution curves. The last question addresses mathematical appreciation. Each question relates back to the learning level at which the material is learned during the Zombie Lab (see Appendix B for additional discussion).

\subsection{Learning Assessment}

The 52 ODE students completed a test before the lab activity and then again after the lab to assess learning. The test was initially given the first day of the lab and then given again the day after the lab was completed. There was no other discussion of the test nor its content. Additionally, none of the content of the test was directly related to the lab. While one of the main foci of the lab is to achieve learning objectives at higher learning levels, the learning assessment also contained a few items geared towards lower learning levels (see Appendix A for test items). Student responses improved uniformly across test items 7 .

In undergraduate mathematics, students rarely have the opportunity to interact with data and create their own models. The Microsoft, data manipulation and rabbit test items (numbers 1,2 and 3 respectively) from the pre- and post-test measure various aspects of how well students are able to create a mechanistic model from data at a number of learning levels (construct-a-concept,discover-a-relation, creativethinking and comprehension-and-communication). Each of these items saw an increase in student performance. 


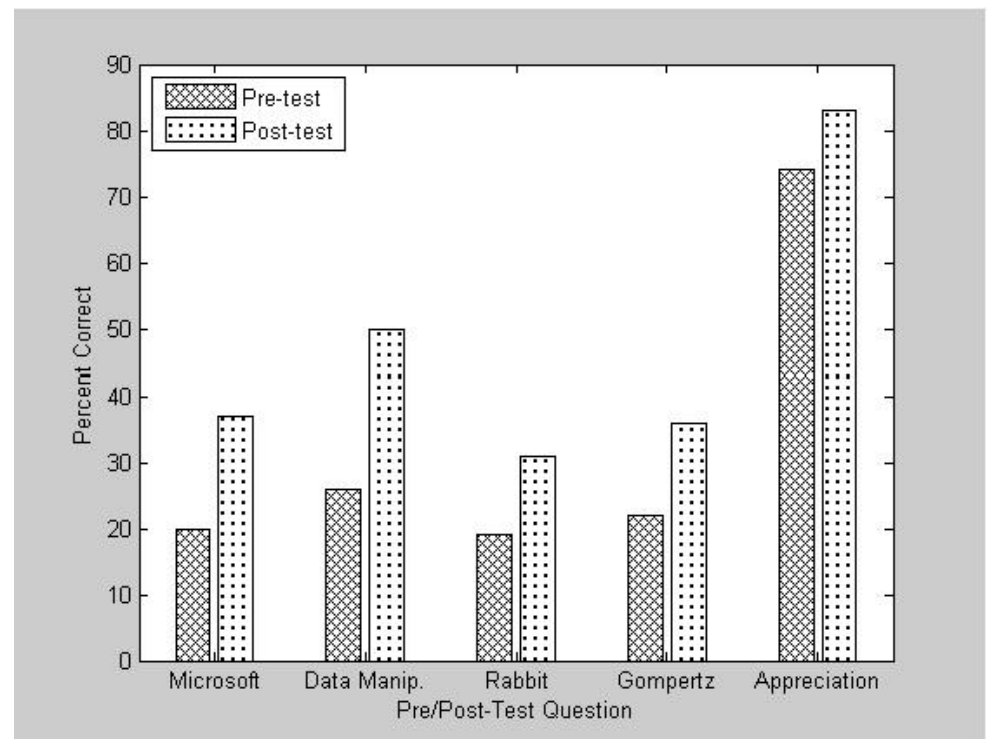

Figure 7. Results from pre-and post-test indicate improvement in each test question. Test questions focused on Zombie Lab learning objectives along with the learning levels at which students were expected to achieve the objectives during the Zombie Lab. Test questions are available in Appendix A with a discussion of test questions in Appendix B.

The Microsoft test item saw an increase from $20 \%$ to $37 \%$ in correct student responses from pre- to post-test with most of the correct responses occurring at the discover-a-relation learning level (e.g., students reason inductively that per capita growth is constant, $\frac{\dot{P}}{P} \approx 3700 \Leftrightarrow$ $\dot{P} \approx 3700 P$, and therefore the population growth must be exponential). On the post-test, a few students answered the prompt at the construct-a-concept learning level by noting how the data looked similar to textbook/internet examples of exponential growth they had encountered while creating a model for the zombie lab.

Acceptable responses to the data manipulation test item (item 2) increased from $26 \%$ to $50 \%$. In nearly every correct response, there was evidence of creative-thinking and comprehension-and-communication learn- 
ing levels. Students consistently used divergent reasoning to produce various ways of arranging population data (creative-thinking learning level) and then effectively communicated how that arrangement could be useful (comprehension-and-communication).

The rabbit test item (item 3) also saw an increase from $19 \%$ to $31 \%$ in correct responses from pre- to post-test. Like the Microsoft test item, in nearly all the correct solutions students reasoned inductively that per capita growth is linear, $\frac{\dot{P}}{P} \approx-0.3 P+3 \Leftrightarrow \dot{P} \approx P(-0.3 P+3)$, and thus the population growth would be approximately logistic(discovera-relation learning level). Additionally, successful students effectively communicated biological descriptions of their model's terms (e.g., intrinsic growth rate is approximately 0.3 and the rabbit populations carrying capacity is approximately 10)(comprehension-and-communication).

Similarly, correct scores increased from $22 \%$ to $36 \%$ on the Gompertz test prompt (item 4). All students who achieved the computation of equilibrium solutions objective did so at the algorithmic skill level by following the procedure presented a couple of class sessions before the start of the lab (i.e., set $\frac{d T}{d t}=0$ and solve for $T$ ). The solution curves objective of the Gompertz test was accomplished at the comprehensionand-communication learning level by accurately describing the dynamics of the equation through the sketching of solution curves.

The final test item, regarding students opinions about the utility of mathematics in the study of disease, also saw a $74 \%$ to $83 \%$ increase in students' scoring from pre- to post-test. Most of the increase from preto post-test is due to students shifting their response from "Mathematics is useful to some scientists in solving problems of limited use to a few scientists working in theoretical areas" (option b) to "Mathematics is of fundamental importance as the study of disease is a quantitative science" (option a). There were also a handful of students who shifted their response from "Mathematics is irrelevant" (option c) to option b or a. These students accounted for the remainder of the increase in test scores. Perhaps most importantly, no student decreased in appreciation (e.g., shifted answer from option a to option c). 


\section{Discussion/Conclusion}

The results from the Microsoft, data manipulation and rabbit test items suggest that students became more proficient at accomplishing modeling tasks after completing the Zombie Lab. Naturally much learning-bydoing occurred since this was the first time most of the students had ever created their own mathematical models. However, the problem-based approach certainly contributed to many students learning at higher cognition levels. The design of the Zombie Lab creates opportunities for instructor scaffolding, leading to higher student cognition, which was evidenced by both test performance and student responses.

In addition to the pre-and post-test, student's comments regarding the lab were noted throughout the activity. Based on their reactions, students are greatly intrigued the Basic Zombie Game and the Logistic Model used to describe the data. It is satisfying for the students to see how the Logistic Model works with respect to an authentic problem and receive visual feedback regarding its success. Additionally, students expressed that analyzing where the model is failing and hypothesizing how they might fix it gave them greater confidence in correcting their own models in the HvZ portion of the lab.

While students are legitimately interested in the Basic Zombie Game, there is an added level of enthusiasm regarding the HvZ data and the challenge of creating their own model for a phenomenon that many of them experienced first-hand as game players. Students emphasized in their reports how this was the first time they felt responsible for their own learning. As one student said, "I wanted to prove to myself that I can use math to do new things." Some made comments about how learning mathematics "...this way made me understand things I thought I already knew." However, there are also students that feel the instructor is "holding out on them." They feel that, like their textbook problems, the instructor has a solutions manual containing the model that will perfectly describe the data. Even though the opportunity to discover mathematics is foreign to most students, students mentioned a number

of times how "[t]he group work was fun and we liked the freedom to 
figure it out with whatever method we chose." Activities and problems that promote discovery tap into students' innate capability of curiosity which intrinsically motivates students to perform at higher levels.

Students commented about how they can better see how mathematics can be a vital tool in telling a story. They expressed how using mathematics in an authentic data-driven manner kept them focused on both creating a final mechanistic model and the narrative it tells. Additionally, the students talked about how playing the HvZ game or simply having seen people play the game made them feel more comfortable with the task. They felt a "...good understanding of the data since [they] helped to create it." Students also mention how they (re)learned many real-life techniques (least squares approximation, Euler solvers, etc.) that they could see being useful beyond the class. Due to the authentic nature of the activity, mathematics became the principle means to understanding and explaining the $\mathrm{HvZ}$ data.

In addition to showing improvement in a variety of areas, student performance indicated deep thinking during initial problem confrontations on the pre-test, then later more ease and fluency in their application of mathematical procedures and topics on the post-test. This is particularly obvious in the model development questions (the Microsoft and rabbit questions). In the pre-test every student that answered correctly appears to have discovered-the-relationship through inductive reasoning. They worked hard to explain the logical basis for their models while detailing what course of action led to their particular belief and why their proposal constituted a reasonably good model. On the post-test rather than searching and discovering, students used deductive reasoning to determine what model they thought would fit the data and then methodically followed approaches taken in the Zombie Lab to support their claims. It appears that the mathematical language students were searching for on the pre-test was readily available on the post-test.

The most critical result is conceivably the boost in students' level of appreciation. While the $9 \%$ increase from pre- to post-test is smaller, the differential equations classes in which the lab was performed were largely comprised of engineering students - students who were already 
convinced of the utility of mathematics.

In addition to the test scores, the benefits of PBL can be seen in students' feedback. The open nature of the Zombie Lab encouraged students to explore and use any resource or concept. There was no correct answer or model and thus students were free to develop their own conclusions. Within the student responses we see that the students interpreted the driving force(s) behind rise and fall of the zombie population quite differently. Not one of these stories was intrinsically right or wrong. The students were empowered with the freedom to follow their own line of thinking. Since students were able to pursue a model of their own creation, they became more familiar with the mathematics needed to generate their model and thus, were able to use mathematics to communicate and illustrate their hypotheses much more fluently than we typically see in undergraduate courses.

Furthermore, students were willing to seek out and learn techniques that are used by researchers and professionals in order to meet the demands of the task. Mechanistic models were built which both predicted the zombie population and alluded to the driving forces behind the dynamics. Students consistently utilized the entire data set when parameterizing their models (e.g., least-squares minimization) rather than relying on the less formal pick-a-point method seen in the textbook.

The HvZ game, when played on campus, is highly visual and quite engaging. We create a naturally compelling and mathematically rich environment by bringing that excitement into the classroom. Giving students interesting problems enables the teacher to press for greater understanding with little resistance. In the Zombie Lab many of the students were keenly interested in the activity so they would have a leg up on the competition the next time the game was played. They could see the immediate utility of learning about and understanding the data.

While we have tried many data-driven activities, the success of the Zombie Lab leads us to propose some rules-of-thumb for PBL, which we call the PAVE framework. A well-designed activity in which students collect their own data has the following traits: 
- Promote Discovery - open-ended with ample opportunity to connect concepts and explore

- Authentic - an original task driven by student-collected data that uses models/techniques actually used by practitioners

- Visible Success - back-story, data and available knowledge make it intuitively (ideally visually) obvious if solution/model is good

- Engaging - an accessible, original question that fits into a broader storyline

The PAVE guidelines can help instructors design successful PBL for college mathematics classroom.

Achieving objectives at higher learning levels is certainly one of the main targets of PAVE. Activities like the Zombie Lab will promote discovery, inciting multiple approaches and discussions that may diverge greatly from the typical curriculum. Since students work with data they were involved with and understand, they get immediate visual feedback on their success, which helps move the instructor away from the role of judge toward the cognition-increasing roles of coach and collaborator. Since the lab is engaging, students respond positively to scaffolding and are more willing to make their own discoveries and seek out and use authentic mathematical techniques (like modeling, numerical solution techniques and least-squares parameterization). 


\section{REFERENCES}

[1] Baden, M.S. and C.H. Major, 2004. Foundations of Problem-Based Learning. Open University Press, Two Penn Plaza, New York, NY.

[2] Barrows, H.S. and R.M. Tamblyn, 1980. Problem-Based Learning: An Approach to Medical Education. Springer Pub. Co., New York, NY.

[3] Bell, S., 2010. Project-based learning for the 21st century: skills for the future. The Clearing House 83:39-43

[4] Boaler, J., 1998. Open and closed mathematics: student experiences and understandings. Journal for Research on Mathematics Education 29: 41-62.

[5] Boyce, W.E. and R.C. DiPrima, 2009. Elementary Differential Equations and Boundary Value Problems (9th ed.). John Wiley \& Sons, Hoboken, NJ.

[6] Cangelosi, J.S., 2003. Teaching Mathematics in Secondary and Middle School: An Interactive Approach (3rd ed.). Pearson Prentice Hall, Upper Saddle, NJ.

[7] Cselle, G., Retrieved January 28, 2013. Timeline: employee headcounts for Google, Yahoo, and Microsoft. http://blog.gaborcselle.com/2006/02/timeline-employeeheadcounts-for.html.

[8] Powell, J., J. Cangelosi and A. Harris, 1998. Games to teach mathematical modelling. SIAM Review 40: 87-95.

[9] Powell, J. A., B.R. Kohler, J.W. Haefner and J. Bodily, 2012. Carrying biomath education in a leaky bucket. Bulletin of Mathematical Biology 74: 2232-2264.

[10] Snee, R.D., 1993. What's missing in statistical education? American Statistician 47(2): 149-154 
[11] Schoenfeld, A. H., 1985. Mathematical problem solving. Academic Press, New York, NY.

[12] Verhulst, Pierre-François, 1838. Notice sur la loi que la population poursuit dans son accroissement. Correspondance Mathématique et Physique 115-116.

[13] Weed, C., B. Sappington, J. Sklover, J. Quick, J., T. Moorman, B. Beecher and M. Temkin, Retrieved November 30, 2013. What is Humans vs Zombies? http://humansvszombies.org 


\section{Appendix A: Pre-/Post-test}

In order to better assess student learning, tests were administered before and after the lab. Questions from the tests along with the targeted learning levels are listed below. Learning levels as defined in [6], "...determine the manner in which students will mentally interact with the objective's mathematical content once the objective is achieved." Targeted learning levels are not included in the student version of the test.

1. (Discover-a-Relation, Construct-a-Concept) John is studying how some businesses flourish over time. Using the reported per capita employee growth of Microsoft from 1990-2005, John calculates and draws the line of best fit. He then conjectures that Microsoft experienced exponential employee growth over that interval. Explain why you either agree or disagree with John's assessment [7].

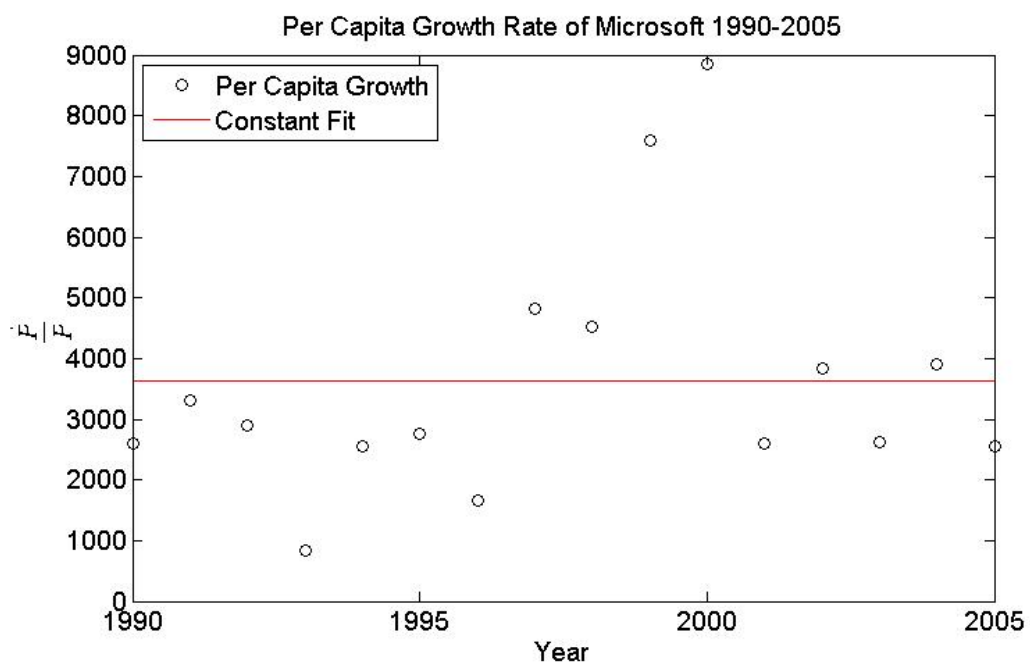

Figure 8. Plot for test item 1 displays percapita growth data of Microsoft employees from 1990 to 2005 along with the line of best fit.

2. (Creative-Thinking, Comprehension-and-Communication) Given data describing the population, $P$ over time, list three different plots (or ways of arranging the data) that may help you develop a model and 
and give reasons for your choices.

3. (Discover-a-Relation, Comprehension-and-Communication) On the virtual farm, the virtual farmer has been simulating a new breed of digital rabbits. His observations are presented in the plot below. Use the virtual farmer's data to develop a population model. Be sure to give a biological description of any parameters you may introduce along with a description of their units.

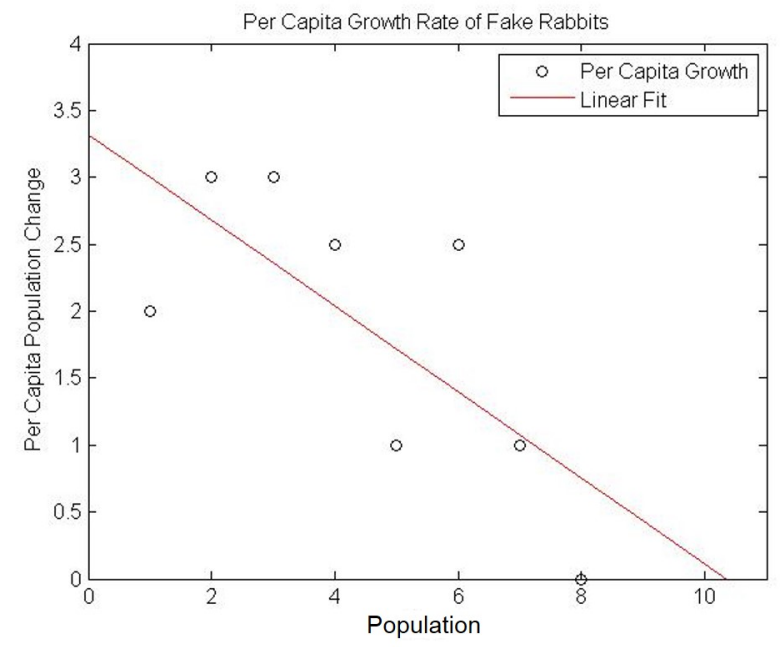

Figure 9. Plot for test item 3. Percapita growth of a fictional rabbit population is displayed along with the line of best fit.

4. (Algorithmic Skill) Tumors are cellular populations, $T$, growing in a confined space where the availability of nutrients is limited. The Gompertz curve has been successfully fit to data of growth of tumors. The Gompertz differential equation is of the form

$$
\frac{d T}{d t}=r T \log \left(\frac{K}{T}\right)
$$

where $r$ is the intrinsic growth rate. What are the equilibrium solutions of the Gompertz equation?

(Comprehension-and-Communication) Sketch a few solution curves that illustrate the dynamics of the model. 
5. (Appreciation) In your opinion what is the role of mathematics in the study of disease? Mathematics is (circle one)

a. of fundamental importance as the study of disease is a quantitative science

b. useful to some scientists in solving problems of limited use to a few scientists working in theoretical areas

c. irrelevant

Write a sentence or two explaining your choice above.

\section{Appendix B: Pre-/Post-test Alignment with Learning Levels}

The first test item is about the number of Microsoft employees from 1990 and 2005. A plot of the per capita employee population growth as well as the line of best fit (a constant function) are provided. The student must determine whether the data and the given line of best fit indicate that the number of Microsoft employees was growing exponential from 1990 to 2005. The test item targets construct-a-concept or discover-arelation learning levels since the student must either distinguish that the given data is indeed an example of exponential growth (constructa-concept) or the student must discover that the data is exponential (discover-a-relation).

Since creating models from data frequently involves the manipulation of data in order to develop model ideas, the second test item asks students to "...list three different plots (or ways of arranging the data) that may help you develop a model and and give reasons for your choices." The creative-thinking learning level is the primary target, but the question also asks students to interact with the material at the comprehension-and-communication learning level since they must also communicate how the arrangement may be useful in model creation.

The initial part of the third test item is similar to the first, except its focus is on a fictitious rabbit population. The students are given the per capita growth of the rabbit population and the line of best fit (a decreasing line) and asked to create a model from the data. Ideally, the student

would either construct the model at the discover-a-relation learning level 
by discovering how the given plot leads to the logistic model or alternatively, the student may construct the model at the construct-a-concept learning level by distinguishing the data as an example of logistic growth. The second portion of the test item addresses our aim of creating biologically meaningful models. In addition to creating a model, the student is also expected to give a mechanistic description of terms in their model for the rabbit population (comprehension-and-communication learning level).

Test item four targets is more in line with a typical differential equations course. It asks students to compute equilibrium solutions of a tumor growth model, Gompertz equation $\frac{d T}{d t}=r T \log \left(\frac{K}{T}\right)$, where $T$ is the cellular population, $r$ is the intrinsic growth rate and $K$ is the carrying capacity. The student would find the equilibrium solutions at the algorithmic skill learning level by remembering and properly executing the steps to find the equilibrium solutions. Additionally, item four directs students to draw a few representative solution curves to the Gompertz equation. The test item targets the comprehension-and-communication learning level since the student must communicate through the sketch that the curves drawn indeed represent the family of solutions to the Gompertz equation.

Given that most students in differential equations classes are aiming for a profession that tends to be math heavy, we wanted to determine whether participating in the Zombie Lab, a more credible experience, would impact their perception of the utility of mathematics in disease study. Hence, the fifth test item specifically asks students' opinion of the role of mathematics in epidemiology. The test item is situated firmly at the appreciation learning level since the student must indicate whether the mathematical content has value. 